\title{
CB Research fuare \\ Development and Evaluation of Models to Predict Readmission or Death After Discharge from Intensive Care
}

\author{
Jamie M Boyd \\ University of Calgary and Alberta Health Services \\ Matthew T James \\ University of Calgary and Alberta Health Services \\ Danny J Zuege \\ University of Calgary and Alberta Health Services \\ Henry Thomas Stelfox ( $\nabla$ tstelfox@ucalgary.ca) \\ University of Calgary and Alberta Health Services
}

\section{Research Article}

Keywords: intensive care units, critical care, death, patient readmission, systematic review, artificial intelligence

Posted Date: September 9th, 2021

DOI: https://doi.org/10.21203/rs.3.rs-841701/v1

License: () (i) This work is licensed under a Creative Commons Attribution 4.0 International License. Read Full License 


\section{Abstract \\ Background}

Patients being discharged from the intensive care unit (ICU) have variable risks of subsequent readmission or death; however, there is limited understanding of how to predict individual patient risk. We sought to derive risk prediction models for ICU readmission or death after ICU discharge to guide clinician decision-making.

\section{Methods}

Systematic review and meta-analysis to identify risk factors. Development and validation of risk prediction models using two retrospective cohorts of patients discharged alive from medical-surgical ICUs ( $n=3$ ICUs, $n=11$,291 patients; $n=14$ ICUs, $n=11$,400 patients). Models were developed using literature and data-derived weighted coefficients.

\section{Results}

Sixteen variables identified from the systematic review were used to develop four risk prediction models. In the validation cohort there were 795 (7\%) patients who were re-admitted to ICU and 703 (7\%) patients who died after ICU discharge. The area under the curve (AUROC) for ICU readmission for the literature $(0.615$ [95\% Cl: $0.593,0.637])$ and data $(0.652$ [95\% Cl: $0.631,0.674])$ weighted models showed poor discrimination. The AUROC for death after ICU discharge for the literature $(0.708$ [95\% Cl: $0.687,0.728])$ and local data weighted $(0.752$ [ $95 \% \mathrm{Cl}: 0.733,0.770])$ models showed good discrimination. The negative predictive values for ICU readmission and death after ICU discharge ranged from $94 \%-98 \%$.

\section{Conclusions}

Identifying risk factors and weighting coefficients using systematic review and meta-analysis to develop prediction models is feasible and can identify patients at low risk of ICU readmission or death after ICU discharge.

\section{Introduction}

Transitions of care are high-risk periods in healthcare delivery.[1, 2] This is particularly true for patients recovering from critical illness as they transfer from the intensive care unit (ICU) to a less-acute hospital ward.[3-5] Although these patients have begun to regain their health and no longer require life-sustaining technologies,[4] there are risks associated with discharge to a more resource limited environment (which usually includes a change in patient-provider ratio), $[6,7]$ including adverse events, ICU readmission and death after ICU discharge.[8-11]

The ICU discharge process begins with the decision to transfer the patient, which requires clinical judgment.[12, 13] Clinical decisionsupport tools can aid clinicians to make better decisions. A limited literature suggests that clinical decision-support tools may help clinicians with ICU discharge decision-making;[14-17] however, most existing tools are limited to a few select risk factors or to one outcome (e.g., ICU readmission).[18, 19] Moreover, the existing published tools have undergone limited validations, employ highly variable risk factors and exhibit mixed degrees of potential clinical utility.[20] We conducted a multi-methods study to develop and evaluate models to predict patient readmission to ICU or death after ICU discharge. Our objective was to derive risk prediction models using a combination of risk factors that have been shown to be consistently associated with readmission to ICU or death after ICU discharge in the literature. The validity of the models was then evaluated in a large independent cohort of critically ill patients.

\section{Methods}

We employed multiple methods over three distinct phases of work to derive and validate individual prediction models for ICU readmission and death after ICU discharge. First, we conducted a systematic review and meta-analysis to identify risk factors previously reported to be consistently associated with ICU readmission or death after discharge from a medical-surgical ICU. Second, we used the risk factors identified to inform the derivation of risk prediction models in a retrospective cohort of patients. Finally, we 
evaluated the operating characteristics (validation) of the derived models in an independent cohort of patients. ICU readmission was defined as a patient with an unplanned readmission to ICU during the index hospitalization. Death after ICU discharge was defined as patients discharged alive from ICU who later died during the index hospitalization. Model development and validation are reported according to the TRIPOD statement.[21]

\section{Identification of Risk Factors}

We conducted a systematic review and meta-analysis in accordance with the Preferred Reporting for Systematic Reviews and MetaAnalyses (PRISMA).[22] The search was conducted on April 1, 2016 and included three term clusters: intensive care units, patient discharge, and ICU readmission/ death after ICU discharge. Two reviewers (JMB, MLP, KJM, RBM) independently reviewed titles and abstracts, and subsequently full text citations, including articles meeting the following criteria: 1) cohort study or controlled trial; 2) original data on human adult ICU patients ( $\geq 18$ years); 3 ) explored one or more risk factors present at ICU discharge; 4) examined ICU readmission and/or in-hospital mortality as an outcome; 5) outcome evaluated after ICU discharge but prior to hospital discharge; and 6) reported summary measure of association (adjusted odds ratio [OR]) or adequate raw data for its calculation. Significant clinical and methodological heterogeneity between studies was anticipated and therefore a meta-analysis of identified risk factors was performed using the DerSimonian and Laird method for random-effects models.[23] The detailed methods of the systematic review are presented in Appendix 1. Risk factors were defined as consistently associated with an outcome if: there were two or more studies examining the risk factors and the $95 \%$ confidence interval $(\mathrm{Cl})$ of the pooled OR did not include the null value. These risk factors were mapped to available data points from the data repository. Operational definitions of candidate risk factors were derived from the authors' definitions of the included studies (see Appendix 2).

\section{Patients}

We identified two distinct cohorts of consecutive adult medical-surgical patients ( $\geq 18$ years) discharged alive from ICUs in Alberta, Canada that used electronic medical record systems during the study period for the derivation (three ICUs incorporating patients admitted between January 1, 2002 and December 31, 2011) and validation (14 ICUs incorporating patients admitted between January 1, 2014 and September 30, 2016) of the risk models. Patients were excluded if the: 1) patient was not discharged from a medical-surgical ICU (e.g., burn ICU); 2) discharge was not to a hospital ward (e.g., discharged directly home); or 3 ) patient was $<18$ years of age. For patients with multiple ICU readmissions within the index hospital admission, or multiple hospital admissions during the study period, only data from the first ICU admission for the first hospitalization was used in the analysis. Patients were identified and data collected from linked clinical and administrative databases previously used for quality improvement and research initiatives (eCritical,[24] Discharge Abstract Database [DAD],[25] Sunrise Clinical Manager [SCM][26]).

\section{Weighting of Risk Scoring Systems}

Two different approaches to model development were used. The Literature weighted method applied a literature-based approach using the beta-coefficients identified through the meta-analysis to weight the selected covariates, The Local data weighted method applied weights derived from including all the covariates selected from the meta-analysis in a logistic regression model in the derivation cohort of patients to determine their coefficients. For both outcomes of interest (ICU Readmission \& death after ICU discharge), the models included all factors consistently associated with risk of the respective outcomes that could be mapped to the administrative datasets. The baseline risk $\left(\beta_{0}\right)$ of the patients included in the derivation cohort was applied to the models for both the Literature weighted and Local data weighted methods. A total of four models were developed using two methods of model construction for testing in the external validation cohort (both literature weighted \& local data weighted ICU readmission models \& death after ICU discharge models).

\section{Statistical Analysis}

Patient and hospital characteristics were explored using descriptive statistics. Using a two-tailed alpha of $5 \%$, we determined the discrimination characteristics for the Literature weighted (model validation) and Local data weighted (model derivation and validation) prediction models using area under the receiver operator characteristic (AUROC) curves.[27, 28] Clinically relevant risk thresholds of ICU readmission and death after ICU discharge $(3 \%, 5 \%, 10 \%)$ were applied to the models to examine performance based on positive and negative predictive values.[18, 29] We assessed model calibration using observed versus expected plots across deciles of predicted risk, and the Hosmer-Lemeshow $X^{2}$ goodness of fit test.[27, 28, 30] Calibration slopes were also evaluated 
for each model during model validation by plotting the observed versus predicted values ( $b>1$ represents over-fitting of the model). $[31,32]$

\section{Results \\ Systematic Review}

The literature search identified 26,897 unique citations from which 832 abstracts were judged to be eligible for full-text review. We identified 65 full-text articles for inclusion in the review (see Appendix 3). The main reasons for citation exclusion during the full text review were studies that: did not include outcome of interest $(n=310)$, were not original research $(n=143)$ or were not related to the ICU discharge process $(n=120)$. Inter-rater agreement for full-text inclusion was good $(K=0.85,95 \% \mathrm{Cl}: 0.56,0.94)$. Appendix 4 presents the study characteristics of the included studies. The forest plots of the random-effects models for ICU readmission and death after ICU discharge are depicted in Appendices 5 and 6.

Risk factors independently associated with ICU readmission included age (years), history of cancer, history of immunosuppression, history of renal disease, history of respiratory disease, Charlson Comorbidity Score $\geq 1$, ICU admission from ward, Eosinopenia, Acute Physiology and Chronic Health Evaluation (APACHE II) score > 9 on admission and ICU length of stay (days) (see Appendix 7). Risk factors independently associated with death after ICU discharge included age (years), male, history of liver disease, duration of hospital stay prior to ICU admission (days), ICU admission from ward, medical admission, APACHE II score > 9 on admission, ICU readmission and night-time discharge (see Appendix 8). Goals of care at ICU discharge was found to be strongly associated with death after ICU discharge; however, owing to the limited availability of data for this variable within the validation cohort this covariate was not included in the final models.

\section{Patient Characteristics and Outcomes}

Appendices 9 and 10 depict the flow of patients in the derivation and validation cohorts, respectively. Of the 18,756 patient records in the derivation cohort, 11,291 were eligible for inclusion in the study. Of the 17,841 patient records in the validation cohort, 11,400 were eligible for inclusion in the study. Table 1 describes the patient characteristics of the derivation and validation cohorts. The typical patient in the derivation cohort had a medical reason for admission (48\%), was male (59\%) with a median age of 58 years (interquartile range [IQR] 43, 71) and an APACHE II score on admission of $16($ IQR 12, 19). The typical patient in the validation cohort had broadly similar characteristics. Overall, $9 \%$ and $7 \%$ of patients discharged alive from ICU were readmitted within the index hospitalization (derivation and validation, respectively), and 7\% of patients discharged alive from ICU (excluding those with ICU readmission) died after ICU but prior to hospital discharge in both the derivation and validation cohorts. 
Patient Characteristics of Derivation and Validation Cohorts

\begin{tabular}{|c|c|c|}
\hline CHARACTERISTIC & Derivation $(n=11291)$ & Validation $(n=11400)$ \\
\hline Male & $6627(59)$ & $6693(59)$ \\
\hline Age, y median (IQR) & $58(43,71)$ & $60(46,71)$ \\
\hline \multicolumn{3}{|l|}{ Comorbidities } \\
\hline History of Cancer & $1542(14)$ & $1300(13)$ \\
\hline History of Immunosuppression & $40(0.4)$ & $41(0.4)$ \\
\hline History of Liver Disease & $308(3)$ & $362(4)$ \\
\hline History of Renal Disease & $1207(11)$ & $1674(15)$ \\
\hline History of Respiratory Disease & $2021(18)$ & $1697(17)$ \\
\hline History of Diabetes & 2116 (19) & $2580(26)$ \\
\hline History of Chronic Heart Disease & $2634(23)$ & $1473(15)$ \\
\hline$\geq 1$ Charlson Comorbidity & $7299(65)$ & $7773(68)$ \\
\hline Charlson Index, median (IQR) & $1(0,3)$ & $1(0,3)$ \\
\hline \multicolumn{3}{|l|}{ Admission Class } \\
\hline Medical & $5416(48)$ & $6024(54)$ \\
\hline Neurocritical & $961(9)$ & $637(6)$ \\
\hline Surgical & $4012(36)$ & $3561(32)$ \\
\hline Trauma & $856(8)$ & $979(9)$ \\
\hline \multicolumn{3}{|l|}{ Location Before ICU Admission } \\
\hline Emergency Department & $4086(36)$ & $4648(41)$ \\
\hline Hospital Ward & $2132(19)$ & $2578(23)$ \\
\hline Operating Room & $2998(27)$ & $2676(23)$ \\
\hline Other & $2065(18)$ & $1492(13)$ \\
\hline APACHE II Score on Admission, median (IQR) & $16(12,19)$ & $18(13,24)$ \\
\hline APACHE $\|>9$ on admission & $9799(87)$ & $10260(90)$ \\
\hline Pre-ICU hospital length of stay, days, median (IQR) & $0(0,1)$ & $0(0,1)$ \\
\hline ICU readmission during index hospitalization & $926(8)$ & $833(7)$ \\
\hline \multicolumn{3}{|l|}{ Interventions Received in ICU } \\
\hline Mechanical Ventilation & $8751(78)$ & $7263(64)$ \\
\hline Mechanical Ventilation Duration, days median (IQR) ${ }^{*}$ & $3(2,7)$ & $2(1,5)$ \\
\hline Continuous Renal Replacement Therapy & $321(3)$ & $477(4)$ \\
\hline Continuous Renal Replacement Therapy Duration, days median (IQR) ${ }^{*}$ & $5(3,9)$ & $4(2,6)$ \\
\hline \multicolumn{3}{|l|}{ Patient Characteristics on ICU Discharge } \\
\hline \multicolumn{3}{|c|}{ *Median duration among those patients receiving mechanical ventilation or continuous renal replacement therapy } \\
\hline${ }^{* *}$ Includes deaths in ICU & & \\
\hline
\end{tabular}




\begin{tabular}{|lll|}
\hline CHARACTERISTIC & Derivation $(\mathbf{n = 1 1 2 9 1 )}$ & Validation $(\mathbf{n}=\mathbf{1 1} \mathbf{4 0 0})$ \\
\hline Eosinopenia at Discharge & $3596(32)$ & $3361(29)$ \\
\hline ICU Length of Stay, days median (IQR) & $3(2,7)$ & $4(2,7)$ \\
\hline Night-time discharge (17:00-07:59) & $4869(43)$ & $4857(43)$ \\
\hline Patient Characteristics on Hospital Discharge & $15(8,31)$ \\
\hline Hospital Length of Stay, days median (IQR) & $17(10,37)$ & $940(8)$ \\
\hline Hospital Mortality ${ }^{* *}$ & $1050(9)$ & $795(7)$ \\
\hline Study Outcomes & & $703(7)$ \\
\hline ICU Readmission & $895(9)$ & $776(7)$ \\
\hline Death after ICU discharge & & \\
\hline *Median duration among those patients receiving mechanical ventilation or continuous renal replacement therapy \\
\hline$* *$ Includes deaths in ICU & & \\
\hline
\end{tabular}

Table 2 outlines the risk factors included in the ICU readmission and death after ICU discharge models, and the distribution of the patients in the derivation and validation cohorts across the selected risk factors. In the derivation cohort, patients who were readmitted to ICU were more often initially admitted from a hospital ward when compared to patients not readmitted to ICU ( $24 \%$ versus $18 \%, p<.001)$. Readmitted patients were also more likely to have one or more Charlson comorbidities $(82 \%$ versus $61 \%, p$ $<.001)$ and an APACHE II score > 9 on admission to ICU $(93 \%$ versus $85 \%, p<.001)$ compared to those patients not readmitted to ICU. Patients who died after ICU discharge, but prior to hospital discharge were more often admitted from a hospital ward (30\% versus $18 \%, \mathrm{p}<.001)$ and to have an APACHE II score $>9$ on admission to ICU $(98 \%$ versus $85 \%, \mathrm{p}<.001)$ compared to those patients discharged alive from hospital. 
Table 2

Distribution of Patients across risk factors for ICU Readmission or Death After ICU Discharge ${ }^{*}$

\begin{tabular}{|c|c|c|c|c|c|c|c|c|c|c|c|c|}
\hline \multirow{5}{*}{$\begin{array}{l}\text { Candidate Risk } \\
\text { Factor }\end{array}$} & \multicolumn{6}{|c|}{ Model Derivation Cohort $(n=11291)$} & \multicolumn{6}{|c|}{ Model Validation Cohort $(n=11400)$} \\
\hline & \multicolumn{3}{|c|}{ ICU Readmission } & \multicolumn{3}{|c|}{ Death after ICU } & \multicolumn{3}{|c|}{ ICU Readmission } & \multicolumn{3}{|c|}{ Death after ICU } \\
\hline & Yes & No & $\begin{array}{l}\mathrm{p}- \\
\text { value }\end{array}$ & Yes & No & $\begin{array}{l}\mathrm{p}- \\
\text { value }\end{array}$ & Yes & No & $\begin{array}{l}\mathrm{p}- \\
\text { value }\end{array}$ & Yes & No & $\begin{array}{l}\text { p- } \\
\text { value }\end{array}$ \\
\hline & $\begin{array}{l}n= \\
895\end{array}$ & $\begin{array}{l}n= \\
9,620\end{array}$ & & $\begin{array}{l}n= \\
776\end{array}$ & $\begin{array}{l}n= \\
9,620\end{array}$ & & $\begin{array}{l}n= \\
795\end{array}$ & $\begin{array}{l}n= \\
9,902\end{array}$ & & $\begin{array}{l}n= \\
703\end{array}$ & $\begin{array}{l}n= \\
9,902\end{array}$ & \\
\hline & (\%) & (\%) & & (\%) & (\%) & & (\%) & (\%) & & (\%) & (\%) & \\
\hline Male & $\begin{array}{l}572 \\
(64)\end{array}$ & $\begin{array}{l}5631 \\
(59)\end{array}$ & .002 & $\begin{array}{l}424 \\
(55)\end{array}$ & $\begin{array}{l}5631 \\
(59)\end{array}$ & .036 & $\begin{array}{l}507 \\
(64)\end{array}$ & $\begin{array}{l}5775 \\
(58)\end{array}$ & .003 & $\begin{array}{l}411 \\
(58)\end{array}$ & $\begin{array}{l}5775 \\
(58)\end{array}$ & .941 \\
\hline Age, y, median (IQR) & $\begin{array}{l}63 \\
(50 \\
73)\end{array}$ & $\begin{array}{l}56 \\
(41, \\
70)\end{array}$ & $<.001$ & $\begin{array}{l}73 \\
(62 \\
80)\end{array}$ & $\begin{array}{l}56 \\
(41, \\
70)\end{array}$ & $<.001$ & $\begin{array}{l}62 \\
(53, \\
71)\end{array}$ & $\begin{array}{l}59 \\
(45, \\
70)\end{array}$ & $<.001$ & $\begin{array}{l}71 \\
(60 \\
80)\end{array}$ & $\begin{array}{l}59 \\
(45, \\
70)\end{array}$ & $<.001$ \\
\hline \multicolumn{13}{|l|}{ Comorbidities } \\
\hline History of Cancer & $\begin{array}{l}153 \\
(17)\end{array}$ & $\begin{array}{l}1233 \\
(13)\end{array}$ & $<.001$ & $\begin{array}{l}156 \\
(20)\end{array}$ & $\begin{array}{l}1233 \\
(13)\end{array}$ & $<.001$ & $\begin{array}{l}124 \\
(19)\end{array}$ & $\begin{array}{l}1056 \\
(12)\end{array}$ & $<.001$ & $\begin{array}{l}120 \\
(19)\end{array}$ & $\begin{array}{l}1056 \\
(12)\end{array}$ & $<.001$ \\
\hline $\begin{array}{l}\text { History of } \\
\text { Immunosuppression }\end{array}$ & $\begin{array}{l}4 \\
(0.5)\end{array}$ & $\begin{array}{l}33 \\
(0.3)\end{array}$ & .388 & $\begin{array}{l}3 \\
(0.4)\end{array}$ & $\begin{array}{l}33 \\
(0.3)\end{array}$ & .510 & $0(0)$ & $\begin{array}{l}36 \\
(0.4)\end{array}$ & .173 & $5(1)$ & $\begin{array}{l}36 \\
(0.4)\end{array}$ & .195 \\
\hline $\begin{array}{l}\text { History of Liver } \\
\text { Disease }\end{array}$ & $\begin{array}{l}61 \\
(7)\end{array}$ & $\begin{array}{l}190 \\
(2)\end{array}$ & $<.001$ & $\begin{array}{l}57 \\
(7)\end{array}$ & $\begin{array}{l}190 \\
(2)\end{array}$ & $<.001$ & $\begin{array}{l}54 \\
(8)\end{array}$ & $\begin{array}{l}241 \\
(3)\end{array}$ & $<.001$ & $\begin{array}{l}67 \\
(11)\end{array}$ & $\begin{array}{l}241 \\
(3)\end{array}$ & $<.001$ \\
\hline $\begin{array}{l}\text { History of Renal } \\
\text { Disease }\end{array}$ & $\begin{array}{l}173 \\
(19)\end{array}$ & $\begin{array}{l}848 \\
(9)\end{array}$ & $<.001$ & $\begin{array}{l}186 \\
(24)\end{array}$ & $\begin{array}{l}848 \\
(9)\end{array}$ & $<.001$ & $\begin{array}{l}189 \\
(24)\end{array}$ & $\begin{array}{l}1286 \\
(13)\end{array}$ & $<.001$ & $\begin{array}{l}199 \\
(28)\end{array}$ & $\begin{array}{l}1286 \\
(13)\end{array}$ & $<.001$ \\
\hline $\begin{array}{l}\text { History of } \\
\text { Respiratory Disease }\end{array}$ & $\begin{array}{l}179 \\
(20)\end{array}$ & $\begin{array}{l}1649 \\
(17)\end{array}$ & .031 & $\begin{array}{l}193 \\
(25)\end{array}$ & $\begin{array}{l}1649 \\
(17)\end{array}$ & $<.001$ & $\begin{array}{l}129 \\
(20)\end{array}$ & $\begin{array}{l}1443 \\
(16)\end{array}$ & .012 & $\begin{array}{l}125 \\
(20)\end{array}$ & $\begin{array}{l}1443 \\
(16)\end{array}$ & .027 \\
\hline History of Diabetes & $\begin{array}{l}226 \\
(25)\end{array}$ & $\begin{array}{l}1684 \\
(18)\end{array}$ & $<.001$ & $\begin{array}{l}206 \\
(27)\end{array}$ & $\begin{array}{l}1684 \\
(18)\end{array}$ & $<.001$ & $\begin{array}{l}187 \\
(29)\end{array}$ & $\begin{array}{l}2185 \\
(25)\end{array}$ & .011 & $\begin{array}{l}208 \\
(33)\end{array}$ & $\begin{array}{l}2185 \\
(25)\end{array}$ & $<.001$ \\
\hline $\begin{array}{l}\text { History of Chronic } \\
\text { Heart Disease }\end{array}$ & $\begin{array}{l}347 \\
(39)\end{array}$ & $\begin{array}{l}1973 \\
(21)\end{array}$ & $<.001$ & $\begin{array}{l}314 \\
(40)\end{array}$ & $\begin{array}{l}1973 \\
(21)\end{array}$ & $<.001$ & $\begin{array}{l}80 \\
(28)\end{array}$ & $\begin{array}{l}1260 \\
(15)\end{array}$ & $<.001$ & $\begin{array}{l}133 \\
(22)\end{array}$ & $\begin{array}{l}1260 \\
(15)\end{array}$ & $<.001$ \\
\hline $\begin{array}{l}\geq 1 \text { Charlson } \\
\text { Comorbidity }\end{array}$ & $\begin{array}{l}734 \\
(82)\end{array}$ & $\begin{array}{l}5855 \\
(61)\end{array}$ & $<.001$ & $\begin{array}{l}710 \\
(91)\end{array}$ & $\begin{array}{l}5855 \\
(61)\end{array}$ & $<.001$ & $\begin{array}{l}662 \\
(83)\end{array}$ & $\begin{array}{l}6498 \\
(66)\end{array}$ & $<.001$ & $\begin{array}{l}613 \\
(87)\end{array}$ & $\begin{array}{l}6498 \\
(66)\end{array}$ & $<.001$ \\
\hline $\begin{array}{l}\text { Charlson Index, } \\
\text { median (IQR) }\end{array}$ & $\begin{array}{l}2(1, \\
4)\end{array}$ & $\begin{array}{l}1(0, \\
2)\end{array}$ & $<.001$ & $\begin{array}{l}3(1, \\
5)\end{array}$ & $\begin{array}{l}1(0, \\
2)\end{array}$ & $<.001$ & $\begin{array}{l}2(1, \\
3)\end{array}$ & $\begin{array}{l}1(0, \\
2)\end{array}$ & $<.001$ & $\begin{array}{l}2(1, \\
4)\end{array}$ & $\begin{array}{l}1(0 \\
2)\end{array}$ & $<.001$ \\
\hline \multicolumn{13}{|l|}{ Admission Class } \\
\hline Medical & $\begin{array}{l}417 \\
(47)\end{array}$ & $\begin{array}{l}4538 \\
(47)\end{array}$ & .008 & $\begin{array}{l}461 \\
(60)\end{array}$ & $\begin{array}{l}4538 \\
(47)\end{array}$ & $<.001$ & $\begin{array}{l}408 \\
(52)\end{array}$ & $\begin{array}{l}5192 \\
(53)\end{array}$ & .040 & $\begin{array}{l}424 \\
(61)\end{array}$ & $\begin{array}{l}5192 \\
(53)\end{array}$ & $<.001$ \\
\hline Neurocritical & $\begin{array}{l}73 \\
(8)\end{array}$ & $\begin{array}{l}801 \\
(8)\end{array}$ & & $\begin{array}{l}87 \\
(11)\end{array}$ & $\begin{array}{l}801 \\
(8)\end{array}$ & & $\begin{array}{l}34 \\
(4)\end{array}$ & $\begin{array}{l}533 \\
(5)\end{array}$ & & $\begin{array}{l}70 \\
(10)\end{array}$ & $\begin{array}{l}533 \\
(5)\end{array}$ & \\
\hline Surgical & $\begin{array}{l}355 \\
(40)\end{array}$ & $\begin{array}{l}3446 \\
(36)\end{array}$ & & $\begin{array}{l}211 \\
(27)\end{array}$ & $\begin{array}{l}3446 \\
(36)\end{array}$ & & $\begin{array}{l}289 \\
(37)\end{array}$ & $\begin{array}{l}3103 \\
(32)\end{array}$ & & $\begin{array}{l}169 \\
(24)\end{array}$ & $\begin{array}{l}3103 \\
(32)\end{array}$ & \\
\hline Trauma & $\begin{array}{l}48 \\
(5)\end{array}$ & $\begin{array}{l}793 \\
(8)\end{array}$ & & $\begin{array}{l}15 \\
(2)\end{array}$ & $\begin{array}{l}793 \\
(8)\end{array}$ & & $\begin{array}{l}58 \\
(7)\end{array}$ & $\begin{array}{l}891 \\
(9)\end{array}$ & & $\begin{array}{l}30 \\
(4)\end{array}$ & $\begin{array}{l}891 \\
(9)\end{array}$ & \\
\hline \multicolumn{13}{|l|}{$\begin{array}{l}\text { Location Before ICU } \\
\text { Admission }\end{array}$} \\
\hline $\begin{array}{l}\text { Emergency } \\
\text { Department }\end{array}$ & $\begin{array}{l}245 \\
(27)\end{array}$ & $\begin{array}{l}3579 \\
(37)\end{array}$ & $<.001$ & $\begin{array}{l}262 \\
(34)\end{array}$ & $\begin{array}{l}3579 \\
(37)\end{array}$ & $<.001$ & $\begin{array}{l}239 \\
(30)\end{array}$ & $\begin{array}{l}4153 \\
(42)\end{array}$ & $<.001$ & $\begin{array}{l}256 \\
(36)\end{array}$ & $\begin{array}{l}4153 \\
(42)\end{array}$ & $<.001$ \\
\hline
\end{tabular}




\begin{tabular}{|c|c|c|c|c|c|c|c|c|c|c|c|c|}
\hline \multirow[b]{2}{*}{ Hospital Ward } & \multicolumn{6}{|c|}{ Model Derivation Cohort $(n=11291)$} & \multicolumn{6}{|c|}{ Model Validation Cohort $(n=11400)$} \\
\hline & $\begin{array}{l}216 \\
(24)\end{array}$ & $\begin{array}{l}1683 \\
(18)\end{array}$ & & $\begin{array}{l}233 \\
(30)\end{array}$ & $\begin{array}{l}1683 \\
(18)\end{array}$ & & $\begin{array}{l}248 \\
(31)\end{array}$ & $\begin{array}{l}2066 \\
(21)\end{array}$ & & $\begin{array}{l}264 \\
(38)\end{array}$ & $\begin{array}{l}2066 \\
(21)\end{array}$ & \\
\hline Operating Room & $\begin{array}{l}242 \\
(27)\end{array}$ & $\begin{array}{l}2615 \\
(27)\end{array}$ & & $\begin{array}{l}141 \\
(18)\end{array}$ & $\begin{array}{l}2615 \\
(27)\end{array}$ & & $\begin{array}{l}224 \\
(28)\end{array}$ & $\begin{array}{l}2336 \\
(24)\end{array}$ & & $\begin{array}{l}116 \\
(17)\end{array}$ & $\begin{array}{l}2336 \\
(24)\end{array}$ & \\
\hline Other & $\begin{array}{l}192 \\
(21)\end{array}$ & $\begin{array}{l}1733 \\
(18)\end{array}$ & & $\begin{array}{l}140 \\
(18)\end{array}$ & $\begin{array}{l}1733 \\
(18)\end{array}$ & & $\begin{array}{l}84 \\
(11)\end{array}$ & $\begin{array}{l}1341 \\
(14)\end{array}$ & & $\begin{array}{l}67 \\
(10)\end{array}$ & $\begin{array}{l}1341 \\
(14)\end{array}$ & \\
\hline $\begin{array}{l}\text { APACHE II Score on } \\
\text { Admission, median } \\
\text { (IQR) }\end{array}$ & $\begin{array}{l}17 \\
(14 \\
21)\end{array}$ & $\begin{array}{l}15 \\
(11, \\
19)^{\prime}\end{array}$ & $<.001$ & $\begin{array}{l}21 \\
(17, \\
25)\end{array}$ & $\begin{array}{l}15 \\
(11 \\
19)^{\prime}\end{array}$ & $<.001$ & $\begin{array}{l}20 \\
(15, \\
26)\end{array}$ & $\begin{array}{l}17 \\
(13 \\
23)\end{array}$ & $<.001$ & $\begin{array}{l}24 \\
(19 \\
29)^{\prime}\end{array}$ & $\begin{array}{l}17 \\
(13 \\
23)\end{array}$ & $<.001$ \\
\hline $\begin{array}{l}\text { APACHE } \|>9 \text { on } \\
\text { admission }\end{array}$ & $\begin{array}{l}830 \\
(93)\end{array}$ & $\begin{array}{l}8211 \\
(85)\end{array}$ & $<.001$ & $\begin{array}{l}758 \\
(98)\end{array}$ & $\begin{array}{l}8211 \\
(85)\end{array}$ & $<.001$ & $\begin{array}{l}772 \\
(97)\end{array}$ & $\begin{array}{l}8791 \\
(89)\end{array}$ & $<.001$ & $\begin{array}{l}697 \\
(99)\end{array}$ & $\begin{array}{l}8791 \\
(89)\end{array}$ & $<.001$ \\
\hline $\begin{array}{l}\text { Pre-ICU length of } \\
\text { stay, d, median (IQR) }\end{array}$ & $\begin{array}{l}0(0, \\
3)\end{array}$ & $\begin{array}{l}0(0, \\
1)\end{array}$ & $<.001$ & $\begin{array}{l}1(0, \\
6)\end{array}$ & $\begin{array}{l}0(0, \\
1)\end{array}$ & $<.001$ & $\begin{array}{l}0(0, \\
3)\end{array}$ & $\begin{array}{l}0(0, \\
1)\end{array}$ & $<.001$ & $\begin{array}{l}1(0, \\
5)\end{array}$ & $\begin{array}{l}0(0, \\
1)\end{array}$ & $<.001$ \\
\hline $\begin{array}{l}\text { ICU readmission } \\
\text { ever }\end{array}$ & $\begin{array}{l}895 \\
(100)\end{array}$ & $\begin{array}{l}31 \\
(0.3)\end{array}$ & $<.001$ & $0(0)$ & $\begin{array}{l}31 \\
(0.3)\end{array}$ & .167 & $\begin{array}{l}795 \\
(0)\end{array}$ & $\begin{array}{l}35 \\
(0.3)\end{array}$ & $<.001$ & $\begin{array}{l}3 \\
(0.4)\end{array}$ & $\begin{array}{l}35 \\
(0.3)\end{array}$ & .738 \\
\hline \multicolumn{13}{|l|}{$\begin{array}{l}\text { Interventions } \\
\text { Received in ICU }\end{array}$} \\
\hline $\begin{array}{l}\text { Mechanical } \\
\text { Ventilation }\end{array}$ & $\begin{array}{l}746 \\
(83)\end{array}$ & $\begin{array}{l}7378 \\
(77)\end{array}$ & $<.001$ & $\begin{array}{l}627 \\
(81)\end{array}$ & $\begin{array}{l}7378 \\
(77)\end{array}$ & .009 & $\begin{array}{l}555 \\
(70)\end{array}$ & $\begin{array}{l}6235 \\
(63)\end{array}$ & $<.001$ & $\begin{array}{l}473 \\
(67)\end{array}$ & $\begin{array}{l}6235 \\
(63)\end{array}$ & .022 \\
\hline $\begin{array}{l}\text { Mechanical } \\
\text { Ventilation, d } \\
\text { median (IQR) }\end{array}$ & $\begin{array}{l}4(3, \\
9)\end{array}$ & $\begin{array}{l}3(2, \\
7)\end{array}$ & $<.001$ & $\begin{array}{l}5(3, \\
10)\end{array}$ & $\begin{array}{l}3(2, \\
7)\end{array}$ & $<.001$ & $\begin{array}{l}2(1, \\
5)\end{array}$ & $\begin{array}{l}2(1, \\
5)\end{array}$ & $<.001$ & $\begin{array}{l}4(1, \\
8)\end{array}$ & $\begin{array}{l}2(1, \\
5)\end{array}$ & $<.001$ \\
\hline $\begin{array}{l}\text { Continuous Renal } \\
\text { Replacement } \\
\text { Therapy }\end{array}$ & $\begin{array}{l}63 \\
(7)\end{array}$ & $\begin{array}{l}203 \\
(2)\end{array}$ & $<.001$ & $\begin{array}{l}55 \\
(7)\end{array}$ & $\begin{array}{l}203 \\
(2)\end{array}$ & $<.001$ & $\begin{array}{l}59 \\
(7)\end{array}$ & $\begin{array}{l}370 \\
(4)\end{array}$ & $<.001$ & $\begin{array}{l}48 \\
(7)\end{array}$ & $\begin{array}{l}370 \\
(4)\end{array}$ & $<.001$ \\
\hline $\begin{array}{l}\text { Continuous Renal } \\
\text { Replacement } \\
\text { Therapy, d median } \\
\text { (IQR) }\end{array}$ & $\begin{array}{l}5(3 \\
14)\end{array}$ & $\begin{array}{l}5(3, \\
9)\end{array}$ & .467 & $\begin{array}{l}5(3, \\
10)\end{array}$ & $\begin{array}{l}5(3, \\
9)\end{array}$ & .607 & $\begin{array}{l}4(2 \\
6)\end{array}$ & $\begin{array}{l}4(2 \\
6)\end{array}$ & .953 & $\begin{array}{l}4(2 \\
6)\end{array}$ & $\begin{array}{l}3(2 \\
6)\end{array}$ & .377 \\
\hline \multicolumn{13}{|l|}{$\begin{array}{l}\text { Patient } \\
\text { Characteristics on } \\
\text { ICU Discharge }\end{array}$} \\
\hline $\begin{array}{l}\text { Eosinopenia at } \\
\text { Discharge }\end{array}$ & $\begin{array}{l}279 \\
(31)\end{array}$ & $\begin{array}{l}3047 \\
(32)\end{array}$ & .758 & $\begin{array}{l}270 \\
(35)\end{array}$ & $\begin{array}{l}3047 \\
(32)\end{array}$ & .073 & $\begin{array}{l}227 \\
(29)\end{array}$ & $\begin{array}{l}2901 \\
(29)\end{array}$ & .657 & $\begin{array}{l}233 \\
(33)\end{array}$ & $\begin{array}{l}2901 \\
(29)\end{array}$ & .031 \\
\hline $\begin{array}{l}\text { ICU Length of Stay, } \\
\text { d median (IQR) }\end{array}$ & $\begin{array}{l}4(2, \\
10)\end{array}$ & $\begin{array}{l}3(1, \\
7)\end{array}$ & $<.001$ & $\begin{array}{l}5(2, \\
10)\end{array}$ & $\begin{array}{l}3(1, \\
7)\end{array}$ & $<.001$ & $\begin{array}{l}4(2, \\
9)\end{array}$ & $\begin{array}{l}4(2, \\
7)\end{array}$ & $<.001$ & $\begin{array}{l}5(3, \\
10)\end{array}$ & $\begin{array}{l}4(2 \\
7)\end{array}$ & $<.001$ \\
\hline $\begin{array}{l}\text { Night-time } \\
\text { discharge (17:00- } \\
07: 59)\end{array}$ & $\begin{array}{l}428 \\
(48)\end{array}$ & $\begin{array}{l}4052 \\
(42)\end{array}$ & .001 & $\begin{array}{l}389 \\
(50)\end{array}$ & $\begin{array}{l}4052 \\
(42)\end{array}$ & $<.001$ & $\begin{array}{l}365 \\
(46)\end{array}$ & $\begin{array}{l}4165 \\
(42)\end{array}$ & .035 & $\begin{array}{l}327 \\
(47)\end{array}$ & $\begin{array}{l}4165 \\
(42)\end{array}$ & .021 \\
\hline \multicolumn{13}{|l|}{$\begin{array}{l}\text { Patient } \\
\text { Characteristics on } \\
\text { Hospital Discharge }\end{array}$} \\
\hline $\begin{array}{l}\text { Hospital Length of } \\
\text { Stay, d median (IQR) }\end{array}$ & $\begin{array}{l}42 \\
(23 \\
75)\end{array}$ & $\begin{array}{l}16(9 \\
33)\end{array}$ & $<.001$ & $\begin{array}{l}19 \\
(10 \\
40)\end{array}$ & $\begin{array}{l}16(9 \\
33)\end{array}$ & .002 & $\begin{array}{l}38 \\
(21, \\
70)\end{array}$ & $\begin{array}{l}14(8, \\
28)\end{array}$ & $<.001$ & $\begin{array}{l}20 \\
(11, \\
38)^{\prime}\end{array}$ & $\begin{array}{l}14(8, \\
28)\end{array}$ & $<.001$ \\
\hline \multicolumn{13}{|l|}{ Study Outcomes } \\
\hline ICU Readmission & $\begin{array}{l}895 \\
(100)\end{array}$ & $0(0)$ & & - & - & & $\begin{array}{l}795 \\
(100)\end{array}$ & $0(0)$ & & - & - & \\
\hline
\end{tabular}

*Bolded cells represent risk factors included in prediction models 


\begin{tabular}{|c|c|c|c|c|c|c|c|c|}
\hline \multirow[b]{2}{*}{ Post-ICU Mortality } & \multicolumn{4}{|c|}{ Model Derivation Cohort $(n=11291)$} & \multicolumn{4}{|c|}{ Model Validation Cohort $(n=11400)$} \\
\hline & - & - & $\begin{array}{l}776 \\
(100)\end{array}$ & $0(0)$ & - & - & $\begin{array}{l}703 \\
(100)\end{array}$ & $0(0)$ \\
\hline
\end{tabular}

In the validation cohort, patients who were readmitted to ICU compared to those patients not readmitted, more often had at least one Charlson comorbidity $(83 \%$ versus $66 \%, \mathrm{p}<.001)$ and an APACHE II score $>9$ on admission to ICU $(97 \%$ versus $89 \%, p<.001)$.

Patients who died after ICU discharge, but prior to hospital discharge were more often admitted from a hospital ward (38\% versus $21 \%, p<.001)$ and had an APACHE II score $>9$ on admission to ICU $(97 \%$ versus $89 \%, p<.001)$ compared to those patients discharged alive from hospital.

\section{Risk Scoring System Performance}

\section{Model Derivation}

Appendix 11 describes the operating characteristics of the ICU Readmission and death after ICU discharge models performance in the derivation cohort. The ICU Readmission model (Local data weighted) showed poor predictive accuracy (AUROC: 0.675 [95\%Cl: $0.657,0.693]$ ), whereas the death after ICU discharge model showed good predictive accuracy (AUROC: 0.792 [95\% Cl: 0.777, 0.807).

Model performance was compared using a complete case analysis, imputing missing values (assuming absence of eosinopenia) with no significant difference in model performance.

\section{Model Validation}

Table 3 describes the operating characteristics of the ICU readmission and death after ICU discharge models in the validation cohort. The AUROC for the ICU readmission models for both the Literature weighted (AUROC: 0.615 [95\%Cl: 0.593, 0.637]) and Local data weighted (AUROC: 0.652 [95\%Cl: 0.631, 0.674]) methods showed poor discrimination. The calibration of the Local data weighted model (Fig. 1) was better than for the Literature weighted model (Fig. 2). The AUROC for the death after ICU discharge models for both Literature weighted (AUROC: 0.708 [95\%Cl: 0.687, 0.728]) and Local data weighted (AUROC: 0.752 [95\% Cl: 0.733, 0.770]) methods showed good discrimination. The calibration of the Local data weighted model (Fig. 3) was better than for to the Literature weighted model (Fig. 4). 
Table 3

ICU Readmission and Death after ICU Discharge Models in the Validation Cohort

\begin{tabular}{|c|c|c|c|c|c|c|}
\hline \multirow[t]{2}{*}{$\begin{array}{l}\text { Literature } \\
\text { weighted }\end{array}$} & \multicolumn{2}{|c|}{$\begin{array}{l}\text { ICU Readmission } \\
\text { ROC: } 0.615 \text { ( } 95 \% \mathrm{Cl:} 0.593,0.637)\end{array}$} & \multicolumn{3}{|c|}{$\begin{array}{l}\text { Death After ICU Discharge } \\
\text { ROC: } 0.708 \text { ( } 95 \% \mathrm{Cl}: 0.687,0.728)\end{array}$} & \multirow[b]{2}{*}{$\begin{array}{l}\text { 3\% Predicted } \\
\text { Probability* }^{*}\end{array}$} \\
\hline & $\begin{array}{l}10 \% \text { Predicted } \\
\text { Probability* }^{*}\end{array}$ & $\begin{array}{l}5 \% \text { Predicted } \\
\text { Probability }^{\star}\end{array}$ & $\begin{array}{l}3 \% \text { Predicted } \\
\text { Probability }^{\star}\end{array}$ & $\begin{array}{l}10 \% \text { Predicted } \\
\text { Probability }^{*}\end{array}$ & $\begin{array}{l}5 \% \text { Predicted } \\
\text { Probability }^{*}\end{array}$ & \\
\hline Sensitivity & $19 \%$ & $86 \%$ & $100 \%$ & $43 \%$ & $79 \%$ & $94 \%$ \\
\hline Specificity & $89 \%$ & $28 \%$ & $0 \%$ & $84 \%$ & $48 \%$ & $22 \%$ \\
\hline $\begin{array}{l}\text { Positive } \\
\text { Predictive } \\
\text { Value }\end{array}$ & $12 \%$ & $8 \%$ & $7 \%$ & $16 \%$ & $10 \%$ & $8 \%$ \\
\hline $\begin{array}{l}\text { Negative } \\
\text { Predictive } \\
\text { Value }\end{array}$ & $94 \%$ & $97 \%$ & - & $95 \%$ & $97 \%$ & $98 \%$ \\
\hline $\begin{array}{l}\text { Correctly } \\
\text { Classified }\end{array}$ & $85 \%$ & $32 \%$ & $7 \%$ & $82 \%$ & $50 \%$ & $27 \%$ \\
\hline \multirow[t]{3}{*}{$\begin{array}{l}\text { Local data } \\
\text { weighted }\end{array}$} & \multicolumn{3}{|c|}{ ICU Readmission } & \multicolumn{3}{|c|}{ Death After ICU Discharge } \\
\hline & \multicolumn{2}{|c|}{ ROC: 0.652 (95\% Cl: 0.631, 0.674) } & & \multicolumn{2}{|c|}{ ROC: 0.752 (95\% Cl: 0.733, 0.770) } & \\
\hline & $\begin{array}{l}\text { 10\% Predicted } \\
\text { Probability }\end{array}$ & $\begin{array}{l}\text { 5\% Predicted } \\
\text { Probability* }\end{array}$ & $\begin{array}{l}3 \% \text { Predicted } \\
\text { Probability }^{*}\end{array}$ & $\begin{array}{l}10 \% \text { Predicted } \\
\text { Probability }^{*}\end{array}$ & $\begin{array}{l}5 \% \text { Predicted } \\
\text { Probability* }\end{array}$ & $\begin{array}{l}3 \% \text { Predicted } \\
\text { Probability }^{*}\end{array}$ \\
\hline Sensitivity & $27 \%$ & $83 \%$ & $97 \%$ & $54 \%$ & $82 \%$ & $92 \%$ \\
\hline Specificity & $86 \%$ & $38 \%$ & $10 \%$ & $81 \%$ & $52 \%$ & $34 \%$ \\
\hline $\begin{array}{l}\text { Positive } \\
\text { Predictive } \\
\text { Value }\end{array}$ & $13 \%$ & $9 \%$ & $7 \%$ & $17 \%$ & $11 \%$ & $9 \%$ \\
\hline $\begin{array}{l}\text { Negative } \\
\text { Predictive } \\
\text { Value }\end{array}$ & $94 \%$ & $97 \%$ & $98 \%$ & $96 \%$ & $98 \%$ & $98 \%$ \\
\hline $\begin{array}{l}\text { Correctly } \\
\text { Classified }\end{array}$ & $82 \%$ & $41 \%$ & $16 \%$ & $79 \%$ & $54 \%$ & $38 \%$ \\
\hline
\end{tabular}

\section{Discussion}

Four prediction models for ICU readmission $(n=2)$ and death after ICU discharge $(n=2)$ were developed using two approaches to model building: Literature weighted and Local data weighted approaches. The prediction models for ICU readmission showed limited discrimination whereas the death after ICU discharge models showed considerably better discrimination. Both sets of models had high negative predictive values. These findings suggest that the use of systematic review to identify risk factors, and meta-analysis to derive variable weights for development of clinical prediction models in critical care is feasible and produces similar results to data derived models. Further evaluation of the clinical application of the derived model for death after ICU discharge is warranted. 
The models developed were primarily able to identify patients at low risk of ICU readmission or death after ICU discharge, suggesting they may help identify patients at low risk for these complications and inform early discharge planning.

\section{What are the implications of these observations for clinicians, managers and researchers?}

One, there is clearly a need to develop new approaches to predicting ICU readmission. The poor discrimination ability of the Literature weighted and Local data weighted models is consistent with a growing body of evidence that suggests that the primary factors influencing ICU readmission are unknown.[33] In fact, Brown et al. have suggested that most readmissions to ICU are about as predictable as a random event and therefore not a good indicator of quality of care.[34] Our data models reinforce those observations and the need for novel approaches to predicting ICU readmission.

Two, death after ICU discharge can be predicted with reasonable accuracy. Our findings are consistent with other models in the literature, such as the ICU Discharge Readiness Score by Badawi and Breslow.[35] The implication is that risk prediction models may be able to improve clinical decision-making. Studies of clinical outcomes after ICU discharge have suggested that physicians have limited ability to predict which patients will experience adverse events, ICU readmission or death after ICU discharge.[36, 37] Evaluation of the clinical application of the derived model for death after ICU discharge is warranted.

Three, although the ICU readmission models in this study do not show promise for predicting which patients will be readmitted, they did have good negative predictive values. This suggests that the models may be effective at identifying patients at low risk for readmission. Interestingly the majority of predictors included in the models were fixed at the time of ICU admission (i.e., not modifiable over the course of stay in ICU), raising the possibility that the models could be applied shortly after patient admission to the ICU to identify those for whom early discharge planning may be appropriate. It is possible that machine learning algorithms may be more effective at identifying modifiable predictors to allow clinicians to modify patient risk through clinical decision-making.[38, 39] The death after ICU discharge models could also be used in a similar manner and given their better predictive abilities could be used to guide physician decision-making in considering delaying ICU discharge, or identifying high priority patients for follow-up by ICU outreach teams.[40, 41]

Four, the use of systematic reviews to identify risk factors for development of clinical prediction models in critical care is feasible. The potential value of such an approach is that it takes advantage of aggregate global research to inform model development. Once candidate risk factors have been identified, there is a small improvement in model discrimination when local data are used to determine weights as opposed to measures of association from a meta-analysis. Grams et al used a similar approach of randomeffects modeling of demographics and health factors to predict risk of end-stage renal disease in kidney donor candidates.[42] The practical implication is that healthcare decision-makers can develop and validate prediction models for similar populations elsewhere without necessarily having to locally rederive models, potentially increasing the pace of predictive model development and application.

The findings of this study should be considered in the context of its strengths and limitations. There are three limitations of note. First, the studies identified as part of our systematic review used heterogeneous definitions for the time to the event for outcomes of interest. To minimize bias in the resulting pooled measures of association we needed to exclude some studies. Standardized definitions for ICU readmission and death after ICU discharge could reduce the heterogeneity of pooled measures of association. Second, the study involved data linkages from multiple clinical and administrative data repositories. The analyses are contingent on the variables and quality of data recorded, which for the primary repository for our study has been reported to be of high quality.[24] Finally, this study was performed in a single healthcare system. It is possible that other systems may vary in risk factors associated with either outcome. However, the literature synthesis was not restricted in its scope and the identified risk factors were those commonly associated with either outcome. The novel approach of applying literature-based coefficients to prediction models should be generalizable to other outcomes and settings.

\section{Conclusion}

Using systematic review and meta-analysis to identify consistently associated risk factors to inform risk predictions models is feasible in critical care. The high sensitivity and negative predictive value in the tested models suggest that such models could be

Page $11 / 16$ 
used to facilitate early discharge planning in low-risk patients given the vast majority of predictors are static. Conversely, identifying high-risk patients remains challenging. New strategies, such as machine learning, need to be considered in order to more accurately derive and identify those patients at high risk of ICU readmission and death after ICU discharge.

\section{Abbreviations}

APACHE, Acute Physiology and Chronic Health Evaluation; AUROC, area under the receiver operating characteristics; Cl, Confidence interval; DAD, Discharge Abstract Database; ICU, intensive care unit; IQR, interquartile range; OR, odds ratio; PRISMA, Preferred Reporting for Systematic Reviews and Meta-Analyses; SCM, Sunrise Clinical Manager.

\section{Declarations}

Ethics approval and consent to participate: This study was approved by the Conjoint Research Ethics Board (CHREB) at the University of Calgary (REB16-1263). Consent to participate was waived owing to the study design.

Consent for publication: Not applicable.

Availability of data and materials: The data that support the findings of this study are available from Alberta Health Services but restrictions apply to the availability of these data, which were used under license for the current study, and so are not publicly available.

Authors' contributions:

JMB: Conceptualization, Methodology, Formal analysis, Investigation, Validation, Writing - Original Draft, Writing - Review \& Editing, Visualization, Project Administration

MTJ: Conceptualization, Methodology, Resources, Validation, Writing Review \& Editing, Supervision

DJZ: Conceptualization, Methodology, Resources, Investigation, Validation, Writing Review \& Editing, Supervision

HTS: Conceptualization, Methodology, Resources, Investigation, Validation, Writing Review \& Editing, Supervision

Acknowledgements: We thank the following individuals for their contributions to the study: Melissa L Potestio, Kelly J Mrklas and

Rebecca Brundin-Mather, Shaun Hosein, Nik Bobrovitz, and Simon Berthelot.

Competing interests: The authors declare that they have no competing interests.

Funding: Jamie Boyd was supported by a W21C - Alberta Innovates-Health Solutions (AlHS) Collaborative Research and Innovation Opportunities (CRIO) Health Services Research graduate studentship. Henry Stelfox was supported by an Embedded Clinician Researcher Award from the Canadian Institutes of Health Research.

\section{References}

1. Wagner J, Gabler NB, Ratcliffe SJ, Brown SE, Strom BL, Halpern SD. Outcomes among patients discharged from busy intensive care units. Ann Intern Med. 2013;159(7):447-55. doi: 10.7326/0003-4819-159-7-201310010-00004.

2. Yang JG, Zhang J. Improving the postoperative handover process in the intensive care unit of a tertiary teaching hospital. J Clin Nurs. 2016;25(7-8):1062-72. doi: 10.1111/jocn.13115.

3. Christmas AB, Freeman E, Chisolm A, Fischer PE, Sachdev G, Jacobs DG, et al. Trauma intensive care unit 'bouncebacks': identifying risk factors for unexpected return admission to the intensive care unit. Am Surg. 2014;80(8):778-82.

4. Canadian Critical Care Society. The role of the ICU. https://canadiancriticalcare.org/Patients-\&-Families. Access July 6, 2020.

5. Stelfox HT, Leigh JP, Dodek PM, Turgeon AF, Forster AJ, Lamontagne F, et al. A multi-center prospective cohort study of patient transfers from the intensive care unit to the hospital ward. Intensive Care Med. 2017;43(10):1485-94. doi: 10.1007/s00134-0174910-1.

6. Ward NS, Read R, Afessa B, Kahn JM. Perceived effects of attending physician workload in academic medical intensive care units: a national survey of training program directors. Crit Care Med. 2012;40(2):400-5. doi: 10.1097/CCM.0b013e318232d997.

Page $12 / 16$ 
7. Li P, Boyd JM, Ghali WA, Stelfox HT. Stakeholder Views on Patient Discharge from Intensive Care: Suboptimal Quality and Opportunities for Improvement. Can J Respir. 2015;22(2):109-18. doi: 10.1155/2015/457431.

8. Cooper GS, Sirio CA, Rotondi AJ, Shepardson LB, Rosenthal GE. Are readmissions to the intensive care unit a useful measure of hospital performance? Med Care. 1999;37(4):399-408. doi: 10.1097/00005650-199904000-00009.

9. Rosenberg AL, Watts C. Patients readmitted to ICUs*: a systematic review of risk factors and outcomes. Chest. 2000;118(2):492-502. doi: 10.1378/chest.118.2.492.

10. Kheir F, Shawwa K, Nguyen D, Alraiyes AH, Simeone F, Nielsen ND. A 24-hour postintensive care unit transition-of-care model shortens hospital stay. J Intensive Care Med. 2016;31(9):597-602. doi: 10.1177/0885066615569701.

11. Town JA, Churpek MM, Yuen TC, Huber MT, Kress JP, Edelson DP. Relationship between ICU bed availability, ICU readmission, and cardiac arrest in the general wards. Crit Care Med. 2014;42(9):2037-41. doi: 10.1097/CCM.0000000000000401.

12. Li P, Boyd JM, Ghali WA, Stelfox HT. Stakeholder views regarding patient discharge from intensive care: Suboptimal quality and opportunities for improvement. Can Respir J 2015; 22(2):109-118. doi: 10.1155/2015/457431.

13. Stelfox HT, Lane D, Boyd JM, Taylor S, Perrier L, Straus S, et al. A scoping review of patient discharge from intensive care: opportunities and tools to improve care. Chest. 2015;147(2):317-27. doi: 10.1378/chest.13-2965.

14. Fernandez R, Biagorri F, Navarro G, Artigas A. A modified McCabe score for stratification of patients after intensive care unit discharge: the Sabadell score. Crit Care. 2006;10(6):R179. doi: 10.1186/cc5136.

15. Ofoma UR, Chandra S, Kashyap R, Herasevich V, Ahmed A, Gajic O, et al. Findings from the implementation of a validated readmission predictive tool in the discharge workflow of a medical intensive care unit. An Am Thorac Soc. 2014;11(5):737-43. doi: 10.1513/AnnalsATS.201312-4360C.

16. Kaplan LJ, Maung AA, Lui FY, Barre K, Davis KA. Using the Rothman index to predict early unplanned surgical intensive care unit readmissions. J Trauma Acute Care Surg. 2014;77(1):78-82. doi: 10.1097/TA.0000000000000265.

17. Aegerter P, Boumendil A, Retbi A, Minvielle E, Dervaux B, Guidet B. SAPS II revisited. Intensive Care Med. 2005;31(3):416-23. doi: $10.1007 / \mathrm{s} 00134-005-2557-9$.

18. Hosein F, Roberts DJ, Turin T, Zygun D, Ghali WA, Stelfox HT. A meta-analysis to derive literature-based benchmarks for readmission and hospital mortality after patient discharge from intensive care. Crit Care. 2014;18(6):715. doi: 10.1186/s13054014-0715-6.

19. Livingston BM, MacKirdy FN, Howie JC, Jones R, Norrie JD. Assessment of the performance of five intensive care scoring models within a large Scottish database. Crit Care Med. 2000;28(6):1820-7. doi: 10.1097/00003246-200006000-00023.

20. Hosein FS, Bobrovitz N, Berthelot S, Zygun D, Ghali WA, Stelfox HT. A systematic review of tools for predicting severe adverse events following patient discharge from intensive care units. Crit Care. 2013;17(3):R102. doi: 10.1186/cc12747.

21. Moons KG, Altman DG, Reitsma JB, loannidis JP, Macaskill P, Steyerberg EW, et al. Transparent Reporting of a multivariable prediction model for Individual Prognosis or Diagnosis (TRIPOD): explanation and elaboration. Ann Intern Med. 2015;162(1):W173. doi: 10.7326/M14-0698.

22. Alessandro L, Altman DG, Tetzlaff J, Mulrow C, Gøtzsche PC, loannidis JPA, et al. The PRISMA statement for reporting systematic reviews and meta-analyses of studies that evaluate health care interventions: explanation and elaboration. Ann Intern Med. 2009;151(4):W65-94. doi: 10.7326/0003-4819-151-4-200908180-00136.

23. DerSimonian R, Laird N. Meta-analysis in clinical trials. Control Clin Trials. 1986;7(3):177-88. doi: 10.1016/01972456(86)90046-2.

24. Brundin-Mather R, Soo A, Zuege DJ, Niven DJ, Fiest K, Doig CJ, et al. Secondary EMR data for research and quality improvement: a comparison of manual and electronic data collection from an integrated critical care electronic medical record system. J Crit Care. 2018;47:295-301. doi: 10.1016/j.jcrc.2018.07.021.

25. Discharge Abstract Database Metadata (DAD). Canadian Institute for Health Information, Ottawa. 2020. https://www.cihi.ca/en/discharge-abstract-database-metadata. Accessed July 6, 2020.

26. Chiasson TC, Manns BJ, Stelfox HT. An economic evaluation of venous thromboembolism prophylaxis strategies in critically ill trauma patients at risk of bleeding. PLoS Med. 2009;6(6):e1000098. doi: 10.1371/journal.pmed.1000098.

27. Moons KG, de Groot JA, Bouwmeester W, Vergouwe Y, Mallett S, Altman DG, et al. Critical appraisal and data extraction for systematic reviews of prediction modelling studies: the CHARMS checklist. PLoS Med. 2014;11(10):e1001744. doi:

Page $13 / 16$ 
10.1371/journal.pmed.1001744.

28. de Groot YJ, Lingsma HF, Bakker J, Gommers DA, Steyerberg E, Kompanje EJ. External validation of a prognostic model predicting time of death after withdrawal of life support in neurocritical patients. Crit Care Med. 2012;40(1):233-8. doi: 10.1097/CCM.0b013e31822f0633.

29. Steyerberg EW, Vergouwe Y. Towards better clinical prediction models: seven steps for development and an ABCD for validation. Eur Heart J. 2014;35(29):1925-31. doi: 10.1093/eurheartj/ehu207.

30. Van Calster B, Nieboer D, Vergouwe Y, De Cock B, Pencina MJ, Steyerberg EW. A calibration hierarchy for risk models was defined: from utopia to empirical data. J Clin Epidemol. 2016;74:167-76. doi: 10.1016/j.jclinepi.2015.12.005.

31. Van Houwelingen JC, Le Cessie S. Predictive value of statistical models. Stat Med. 1990;9(11):1303-25. doi: 10.1002/sim.4780091109.

32. Steyerberg EW, Vickers AJ, Cook NR, Gerds T, Gonen M, Obuchowski N, et al. Assessing the performance of prediction models: a framework for traditional and novel measures. Epidemiology. 2010; 21(1):128-38. doi: 10.1097/EDE.0b013e3181c30fb2.

33. Ponzoni CR, Correa TD, Filho RR, Serpa Neto A, Assuncao MSC, Pardini A, et al. Readmission to the intensive care unit: incidence, risk factors, resource use, and outcomes. A retrospective cohort study. Ann Am Thorac Soc. 2017;14(8):1312-9. doi: 10.1513/AnnalsATS.201611-8510C.

34. Brown SES, Ratcliffe SJ, Halpern SD. Assessing the utility of ICU readmissions as a quality metric: an analysis of changes mediated by residency work-hour reforms. Chest. 2015;147(3):626-36. doi: 10.1378/chest.14-1060.

35. Badawi O, Breslow MJ. Readmissions and death after ICU discharge: development and validation of two predictive models. PloS One. 2012; 7(11):e48758. doi: 10.1371/journal.pone.0048758.

36. Detsky ME, Harhay MO, Bayard DF, Delman AM, Buehler AE, Kent SA, et al. Discriminative accuracy of physician and nurse predictions for survival and functional outcomes 6 months after an ICU admission. JAMA. 2017;317(21):2187-95. doi: 10.1001/jama.2017.4078.

37. Sauro KM, Soo A, de Grood C, Yang MMH, Wierstra B, Benoit L, et al. Adverse events after transition from ICU to hospital ward: a multicenter cohort study. Crit Care Med. 2020;48(7):946-53. doi: 10.1097/CCM.0000000000004327.

38. Badawi O, Brennan T, Celi LA, Feng M, Ghassemi M, Ippolito A, et al. Making big data useful for health care: a summary of the inaugural mit critical data conference. JMIR Med Inform. 2014;2(2):e22. doi: 10.2196/medinform.3447.

39. Sanchez-Pinto LN, Luo Y, Churpek MM. Big Data and Data Science in Critical Care. Chest. 2018;154(5):1239-48. doi: 10.1016/j.chest.2018.04.037.

40. Stelfox HT, Bastos J, Niven DJ, Bagshaw SM, Turin TC, Gao S. Critical care transition programs and the risk of readmission or death after discharge from ICU. Intensive Care Med. 2016;42(3):401-10. doi: 10.1007/s00134-015-4173-7.

41. Niven DJ, Bastos JF, Stelfox HT. Critical care transition programs and the risk of readmission or death after discharge from an ICU: a systematic review and meta-analysis. Crit Care Med. 2014;42(1):179-87. doi: 10.1097/CCM.0b013e3182a272c0.

42. Grams ME, Sang Y, Levey AS, Matsushita K, Ballew S, Chang AR, et al. Kidney-failure risk projection for the living kidney-donor candidate. NEJM. 2016;374(5):411-21. doi: 10.1056/NEJMoa1510491.

\section{Figures}




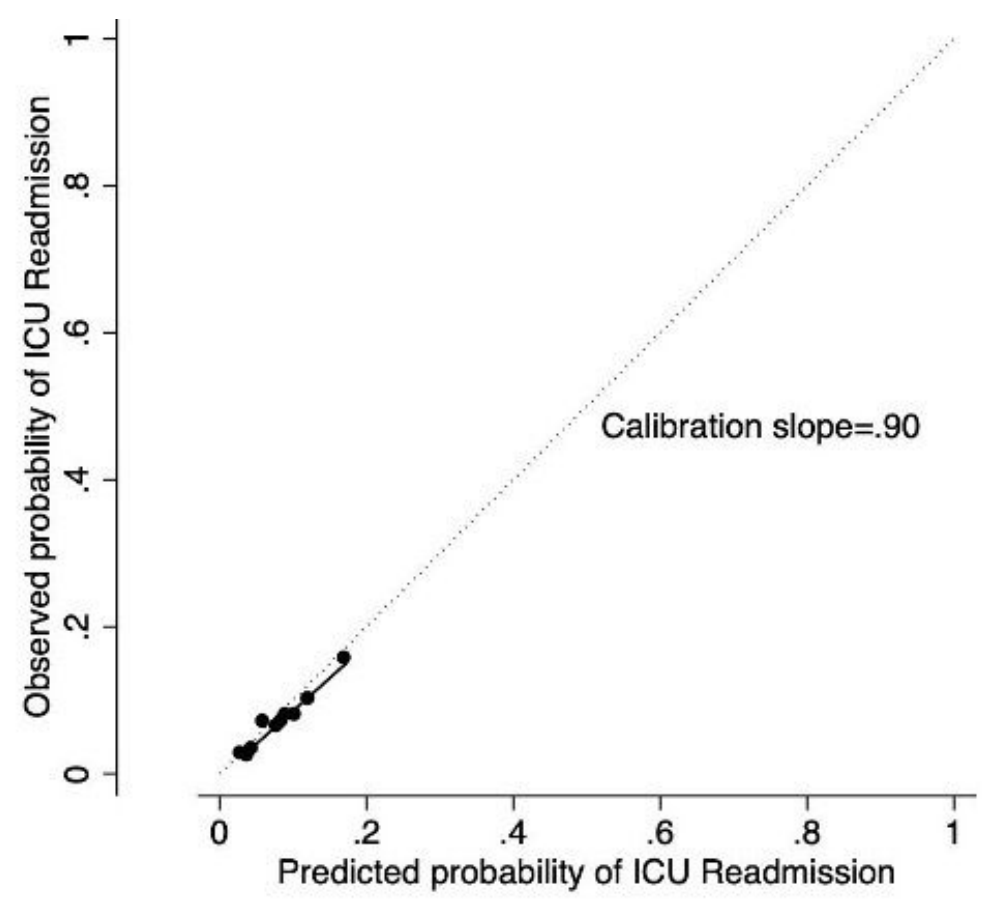

Figure 1

Calibration Slopes - ICU Readmission - Literature Weighted Model Performance

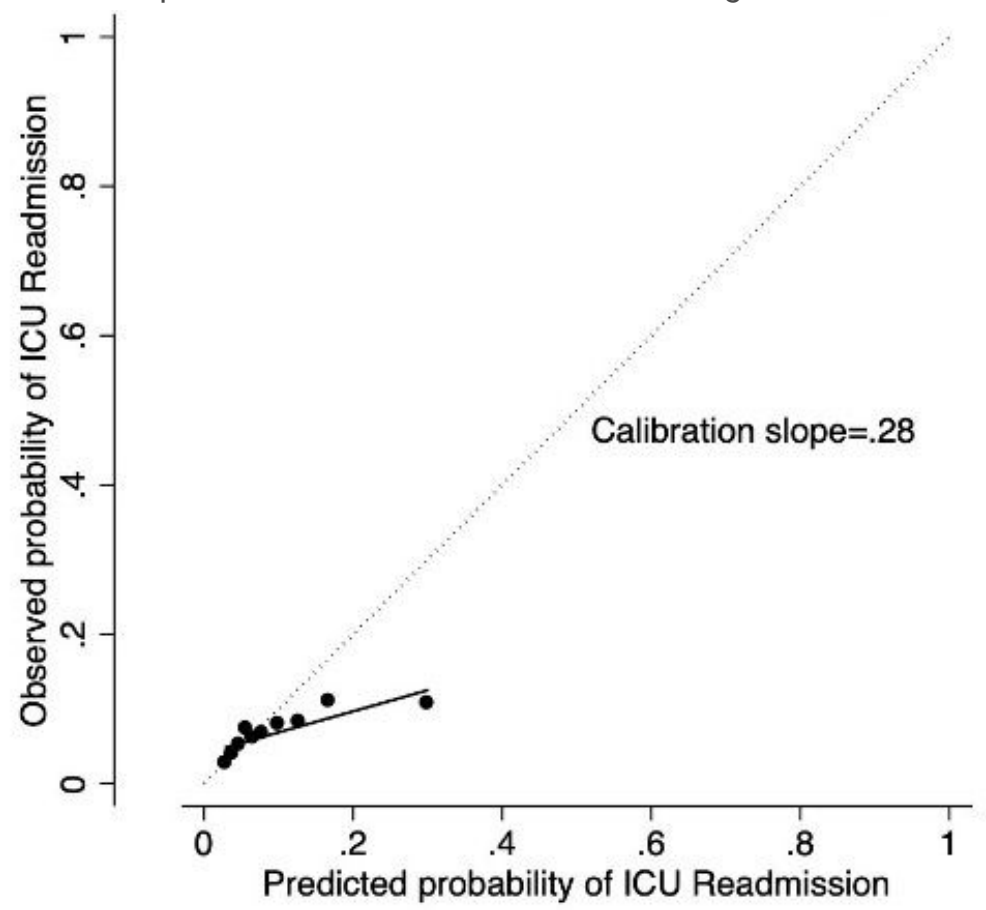

Figure 2

Calibration Slopes - ICU Readmission - Local Data Weighted Model Performance 

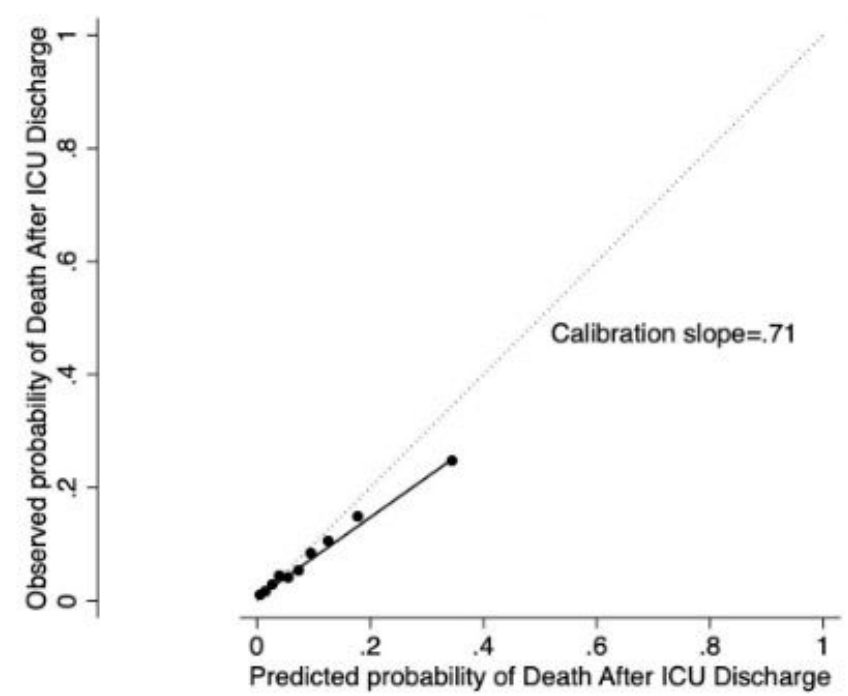

Figure 3

Calibration Slopes - Death After ICU Discharge - Literature Weighted Model Performance

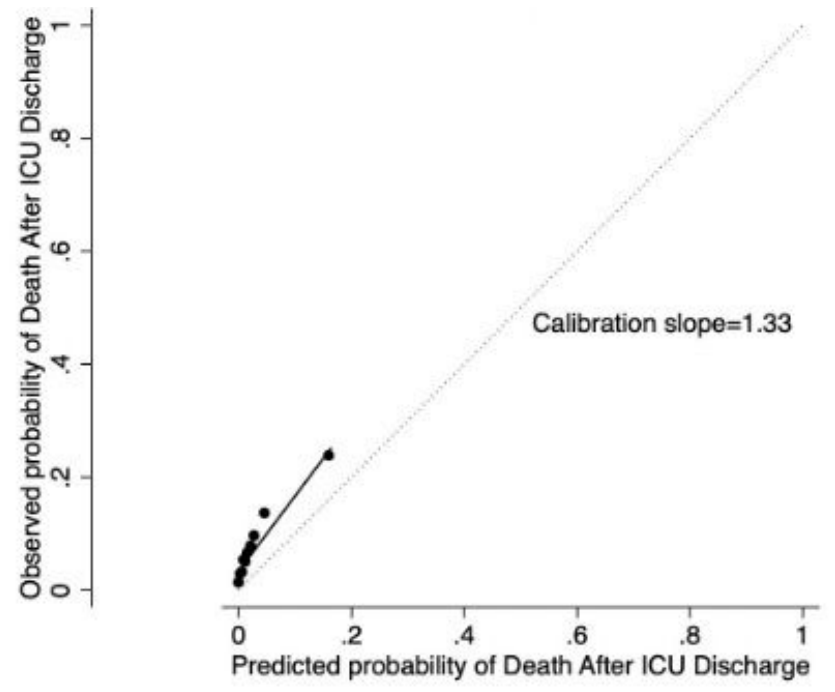

Figure 4

Calibration Slopes - Death After ICU Discharge - Local Data Weighted Model Performance

\section{Supplementary Files}

This is a list of supplementary files associated with this preprint. Click to download.

- AppendicesFileicuriskmodels.docx 